\title{
EVALUATION OF THE RELIABILITY AND THE SENSITIVITY OF QUADRI-LAYERED MO DISK WITH AN AITa ALLOY REFLECTION LAYER BY PCT ENVIRONMENTAL TEST
}

\author{
T. MIYAMOTO, T. ONISHI, S. YAMAMOTO, K. YOSHIKAWA and K. KOGA* \\ Materials Research Laboratories, KOBE, STEEL, LTD., 5-5 Takatsukadai 1-Chome, \\ Nishi-ku, Kobe, Hyogo 651-22, Japan \\ *KOBELCO RESEARCH INSTITUTE, INC, 5-5 Takatsukadai 1-Chome, Nishi-ku, Kobe, Hyogo 651-22, Japan
}

\begin{abstract}
The reliability and the sensitivity of quadri-layered MO disks with an AlTa reflection layer and those with a pure $\mathrm{Al}$ reflection layer were evaluated by the PCT environmental test. In the case of glass substrates CNR of media with a pure $\mathrm{Al}$ reflection layer decreased faster than that with an AlTa reflection layer. Optimum writing power increased steadily, and finally it exceeded the system's maximum laser power for both kinds of media. However, initial writing power of media with an AlTa reflection layer is much lower.Therefore, an AITa reflection layer can maintain higher CNR and offers a large margin. The recording layer in the media with an Al layer degraded more than that with an AlTa reflection layer. This can be attributed to the higher corrosion resistance of an AlTa reflection layer.

$\mathrm{CNR}$ of media fabricated on PC substrates decreased faster than those on glass substrates in the early stage of the PCT test. No significant difference between the two kinds of media was observed in this case.
\end{abstract}

KEYWORDS: MO DISK, RELIABILITY, CNR, PCT TEST, REFLECTION LAYER

\section{INTRODUCTION}

The use of Al alloy films as a reflection layer is known to be effective in reducing the recording power and improving the sensitivity of the quadri-layered MO media [1][2]. The recording sensitivity is strongly affected by the thermal conductivity of the reflection layer used in the media. AlTa and AITi alloy films have low thermal conductivity, and as a result the recording sensitivity is greatly improved. Moreover, the alloys have excellent corrosion resistance, and it is also expected that the reliability of the media will be improved by using these alloy films as a reflection layer instead of a pure $\mathrm{Al}$ layer[2][3].

In this study the reliability and the sensitivity of the media with an A.lTa reflection layer have been evaluated by PCT environmental test while comparing the results with those on media with a pure $\mathrm{Al}$ reflection layer.

\section{EXPERIMENTAL PROCEDURES}

Quadri-layered media with a pure $\mathrm{Al}$ or an $\mathrm{Al}-3$ at.\% Ta alloy reflection layer were fabricated on 3.5 inch grooved glass substrates or PC substrates. Structure of the media is shown in Fig.1.

Three of the media on glass substrate with each reflection layer were exposed to a corrosive environment at $105^{\circ} \mathrm{C}, 1.2 \mathrm{~atm}$ and $100 \% \mathrm{RH}$ for a specific period of time (PCT test hereafter). Three media on
$\mathrm{PC}$ substrate with an $\mathrm{Al}$ reflection layer and four media on PC substrate with an AlTa reflection layer were also tested by exposing them to the same environment.

\begin{tabular}{cc}
\hline UV resin & $(10 \mu \mathrm{m})$ \\
\hline Al or AlTa & $(500 \AA)$ \\
\hline SiN & $(330 \AA)$ \\
\hline Tb-Fe-Co & $(250 \AA)$ \\
\hline SiN & $(980 \AA)$ \\
\hline \multicolumn{2}{c}{ Glass substrate } \\
\hline
\end{tabular}

Fig. 1 Structure of the media

After each PCT test write power dependence of the carrier amplitude, the noise, the second harmonics amplitude and the CNR were measured.

Change of these properties by the PCT test was observed by repeating this procedure. Conditions of the $\mathrm{C} / \mathrm{N}$ measurement are listed in table 1.

Table 1 Conditions of $\mathrm{C} / \mathrm{N}$ measurement

\begin{tabular}{l} 
Frequency $3.7 \mathrm{MHz}$ \\
Duty $33 \%$ \\
Rotation $\quad 1800$ r.p.m. \\
External Field $\quad 250 \mathrm{Oe}$ \\
\hline
\end{tabular}


After the PCT test Polar Kerr loop measurement of the media and a depth profile analysis by Auger spectroscopy were done.

\section{RESULTS AND DISCUSSION}

Results of the PCT test for media on glass substrates

Figure 2 shows change of CNR of the media with an AITa reflection layer together with that of the media with an Al reflection layer by the PCT environmental test. The CNRs shown are the values at the optimum writing power except for those with a longer PCT test for which CNRs at the maximum power of $10 \mathrm{~mW}$ are indicated. They are the average values over three disks of each kind. Bars denote data range for three disks. The CNR of media with a pure $\mathrm{Al}$ reflection layer drops very rapidly compared with that of the media with an AlTa reflection layer.

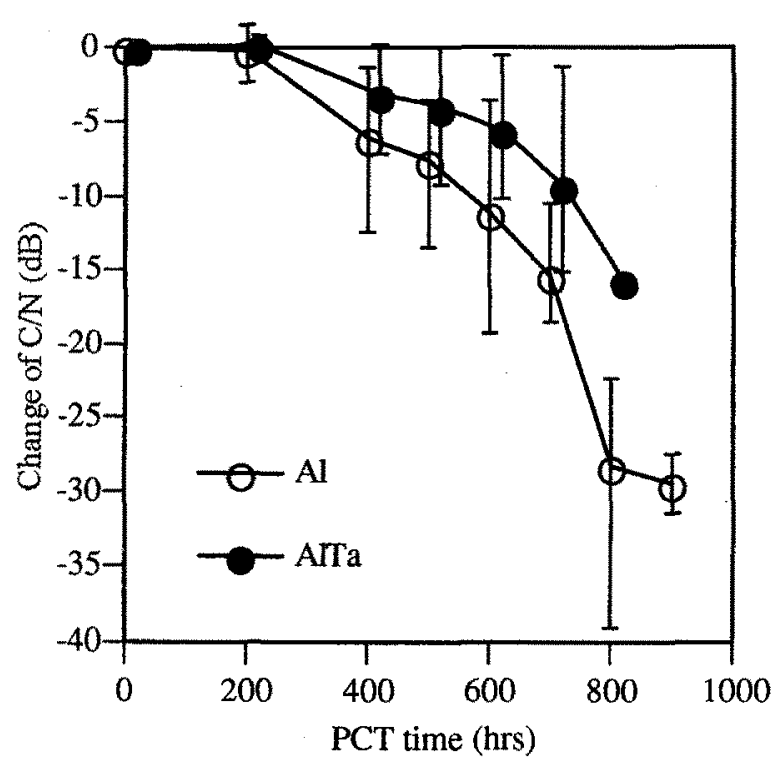

Fig.2 Change of CNR by the PCT test

It has also been observed that the optimum writing power keeps rising throughout the PCT test. The dependence of the average optimum writing power on the PCT time is shown in Fig.3. Figure 4 shows the writing power dependence of the CNR.

The optimum writing power for the media with an AlTa reflection layer is much lower than the case of the Al reflection layer because of the large difference of the thermal conductivity between the films for the reflection layers.

The optimum writing power of both $\mathrm{Al}$ and AITa media increases steadily as the PCT test progresses and finally exceeds the maximum laser power of the system. PCT testing beyond this point

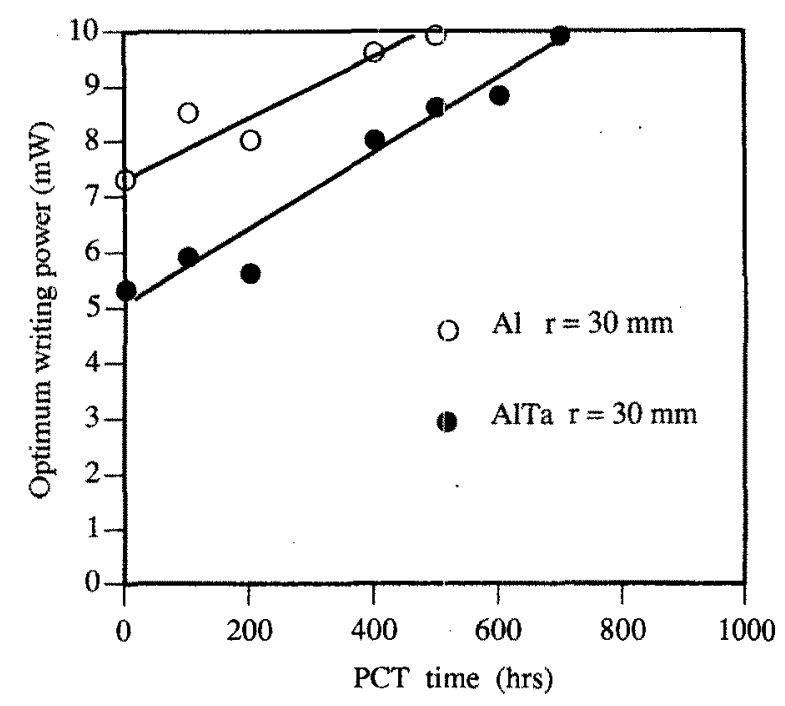

Fig.3 Dependence of the optimum writing power on the PCT time

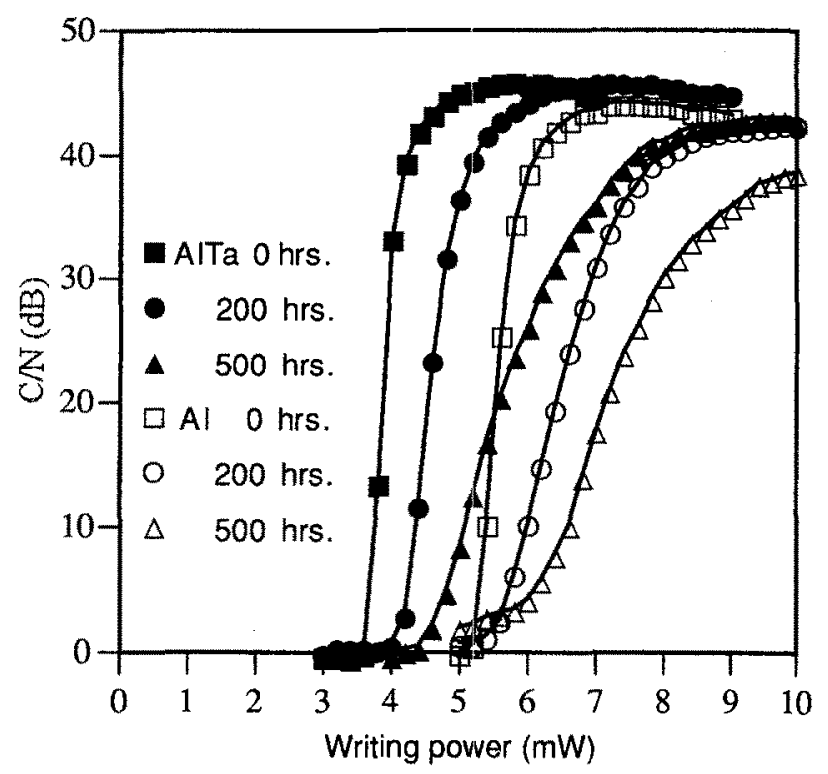

Fig.4 Change of the writing power dependence of the CNR

brings about an abrupt decrease of CNR when measured at the maximum laser power.

The coercivities of media fabricated this time were around $15 \mathrm{KOe}$ or larger before the PCT test. Figure 5 shows Polar Kerr loops of media after 1000 hours' PCT test. Substantial decrease in the coercivity was observed for a medium with an $\mathrm{Al}$ reflection layer.

It has been reported that the oxidization of the RE-TM alloy recording layer brings about a compositional modification resulting from a selective oxidization of the Rare Earth element in the film[4]. 
Figure 6 shows the results of Auger analysis of media after 1000 hours' PCT test. Oxygen content in the recording layer after the PCT test is from 5 to 10 at \% which was less than 1 at\% before the PCT test.

It is concluded that the recording layers have become more TM rich than the initial composition due to the selective oxidization of the RE element.

Most of the media with the AlTa reflection layer stayed at higher coercivity than those of the media with a pure Al reflection layer. The initial decrease of the CNR of the media where the optimum writing power is lower than $10 \mathrm{~mW}$ is supposed to be a result of the oxidization of the recording layer. The rapid decrease of the CNR near the end of the PCT test where the optimum writing laser power is larger than the system's maximum power results from both the lack of the recording laser power and the poor sensitivity of the media with a pure $\mathrm{Al}$ reflection layer.

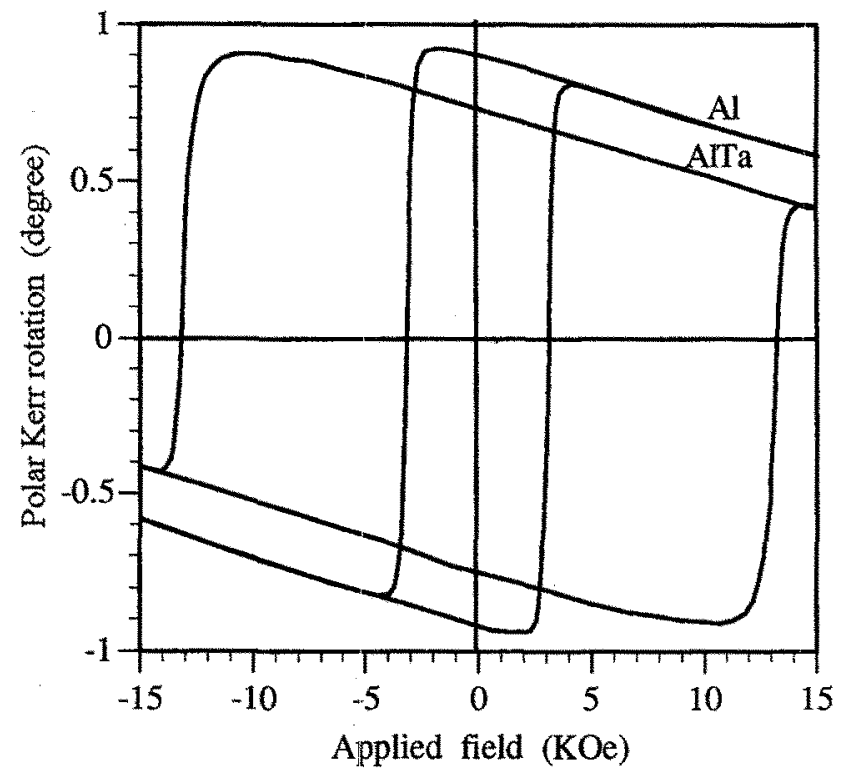

Fig.5 Polar Kerr loops of media after 1000 hours' PCT test

Results of the PCT test for media on PC substrates.

Media fabricated on PC substrates were also subjected to the PCT environmental test. Figure 7 shows change of CNR of the media with an AITa reflection layer together with that of the media with an $\mathrm{Al}$ reflection layer fabricated on PC substrates by the PCT test. They are the average values over disks of each kind.

CNR decreases more rapidly than the case of glass substrates at the early stage of the PCT test. It is also observed that there is not so large a difference in the CNR change between media with an $\mathrm{Al}$ reflection
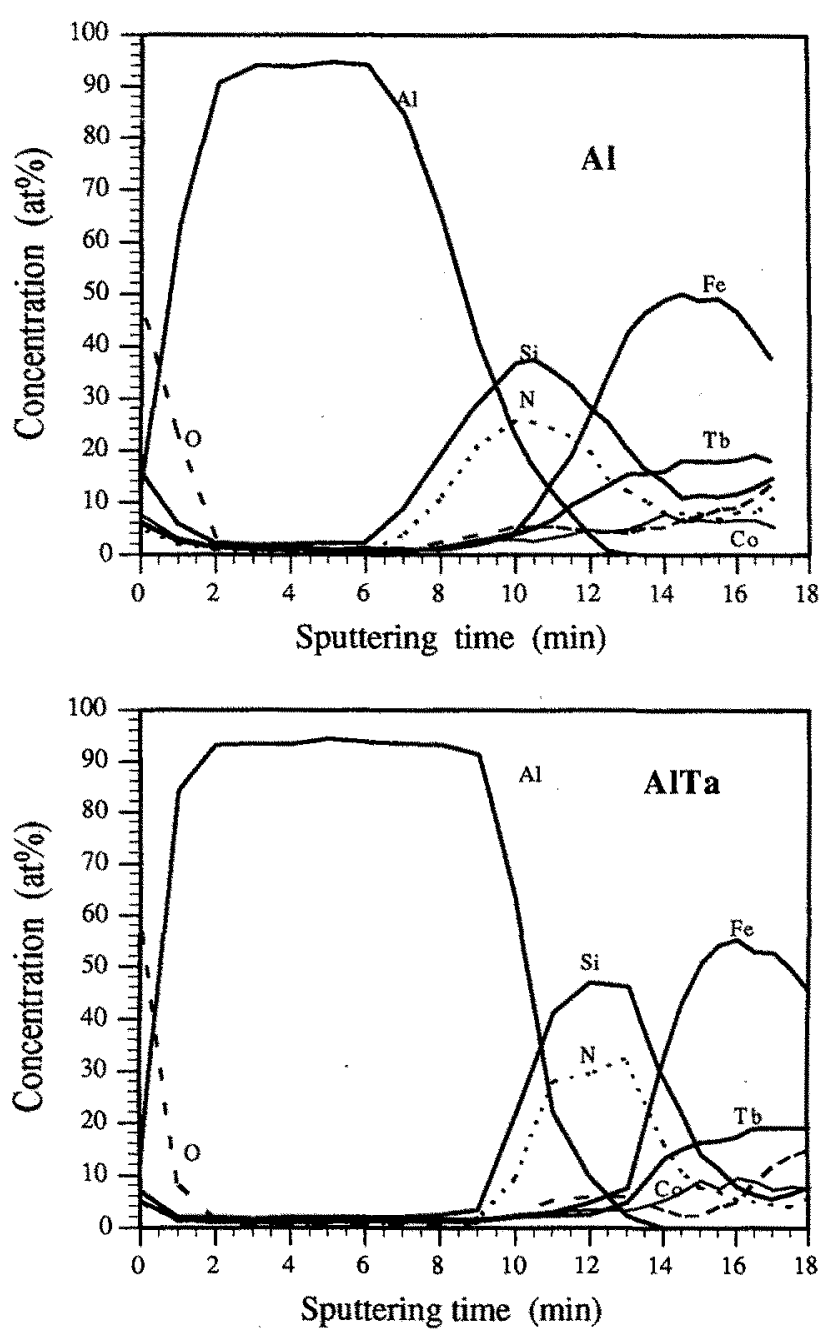

Fig.6 Auger depth profiles of media after the PCT test.

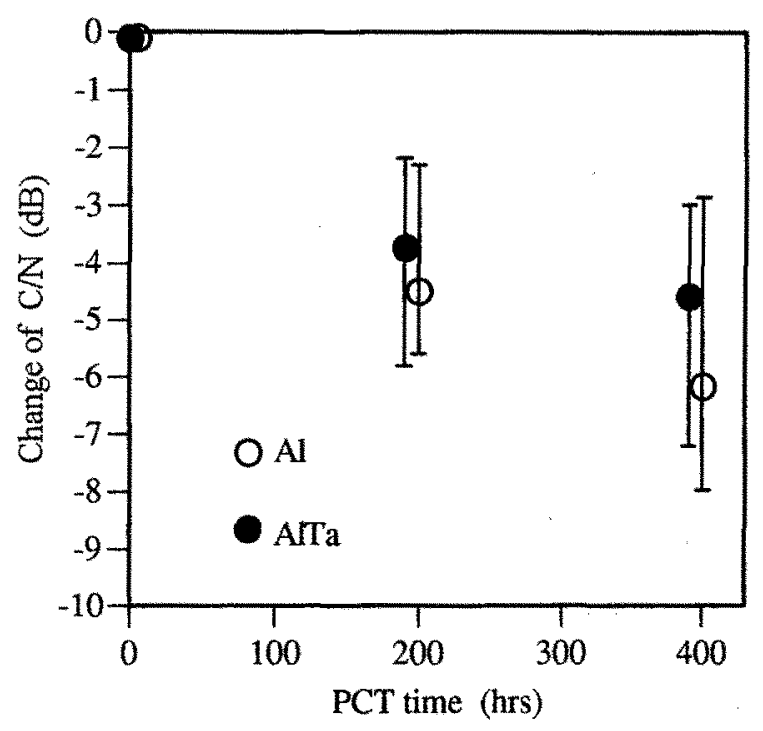

Fig.7 Change of CNR by the PCT test for media fabricated on $\mathrm{PC}$ substrates 
layer and those with an AlTa reflection layer.

Polar Kerr loops before and after the 400 hours' PCT test are shown in Fig.8. Hc of all the media have decreased from about $13 \mathrm{KOe}$ to a value less than $4 \mathrm{KOe}$ by the 400 hours' PCT test irrespective of the reflection layer used. No significant difference in the shape of Kerr loop can be recognized between the two kinds of media.

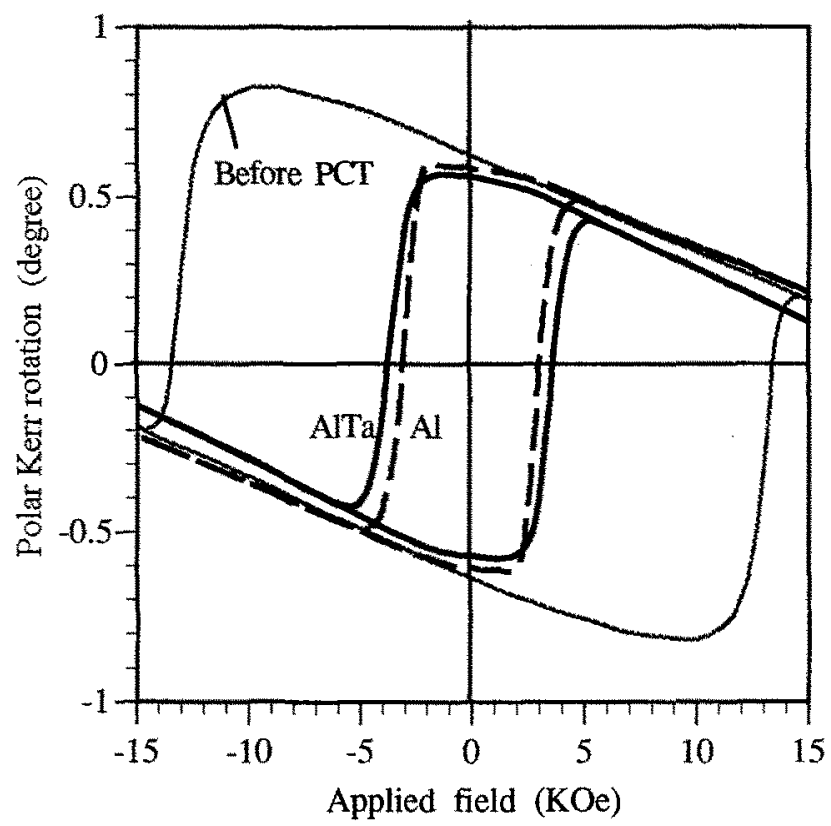

Fig.8 Polar Kerr loops of media on PC substrate after 400 hours' PCT test

Optimum writing power was less than $10 \mathrm{~mW}$ for all the media on PC substrates after 400 hours' PCT test. Dependence of the optimum writing power on the PCT exposure time was similar to that for media on glass substrates except that the initial writing powers were reduced by about $1.5 \mathrm{~mW}$.

The rapid decrease of CNR observed in the case of glass substrates near the end of the PCT test is supposed to be mainly brought about by the effect that the optimum writing power exceeds the system's maximum laser power. On the other hand, CNR of media on PC substrates decreases rapidly at the initial stage before the optimum laser power attains $10 \mathrm{~mW}$. Moreover, the Hcs decreased remarkably already at 400 hours' PCT elapse time.

The difference of the CNR change and the difference of the Kerr loops between media with a different reflection layer were observed only in the case of glass substrate. Oxygen content in the recording layer also differs near the interface between the recording layer and $\mathrm{SiN}$ adjacent to the reflection layer. It is supposed that the extent of deterioration of the recording layer depends on the corrosion resistance of the reflection layer in the case of glass substrates while quality of media fabricated on PC substrates is affected by other factors such as the quality of SiN deposited on the PC substrates, regardless of the corrosion resistance of the reflection layer.

\section{CONCLUSION}

CNR of media with an Al reflection layer decreased faster than that of media with an AlTa reflection layer by the PCT environmental test.

Optimum writing power increased steadily as the PCT test progresses for both kinds of media. However, the initial writing power of the media with an AITa reflection layer is much lower than that of media with a pure $\mathrm{Al}$ reflection layer due to the low thermal conductivity of AlTa alloy films and as a result required writing laser power for media with a pure $\mathrm{Al}$ reflection layer exceeds the system's maximum laser power leading to the rapid decrease of the CNR near the end of the PCT test.

Differences in the deterioration of the recording layer were recognized in the $\mathrm{Hc}$ of the media after the PCT test. Auger analysis shows that the oxygen content in the recording layer near the interface of SIN adjacent to the reflection layer is lower for media with an AlTa reflection layer. It is concluded that media with an AlTa reflection layer have higher environmental durability than those with a pure Al reflection layer in terms of the high sensitivity and the high corrosion resistance.

CNR decreased much faster at the initial stage of the PCT test if fabricated on PC substrates. Significant differences in neither CNR change nor Kerr loop between the two kinds of media after 400 hours' PCT test were observed .

\section{REFERENCES}

[1] T. Kawano, Mitsubishi Kasei R\&D Review, 4(2), 1 (1990)

[2] T. Onishi et al., J. Magn. Soc. Jpn., Vol. 15 No S1, 373 (1991)

[3] T. Onishi et al., J. Magn. Soc. Jpn., Vol. 17 No S1, 106 (1993)

[4] E.E. Marinero et al. IEEE Trans. Magn MAG-23 No 5, 2629 (1987) 